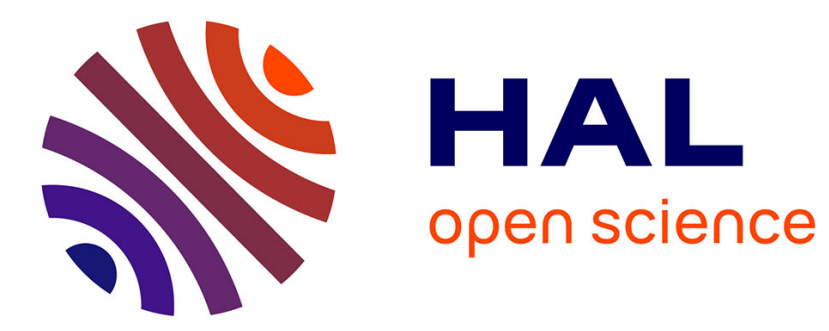

\title{
On One-Rule Grid Semi-Thue Systems
}

\author{
Michel Latteux, Yves Roos
}

\section{To cite this version:}

Michel Latteux, Yves Roos. On One-Rule Grid Semi-Thue Systems. Fundamenta Informaticae, 2012, Words, Graphs, Automata and Languages. Special Issue Honoring the 60th Birthday of Professor Tero Harju, 116 (1-4), pp.189-204. 10.3233/FI-2012-678 . hal-00749289

\section{HAL Id: hal-00749289 \\ https://hal.inria.fr/hal-00749289}

Submitted on 7 Nov 2012

HAL is a multi-disciplinary open access archive for the deposit and dissemination of scientific research documents, whether they are published or not. The documents may come from teaching and research institutions in France or abroad, or from public or private research centers.
L'archive ouverte pluridisciplinaire HAL, est destinée au dépôt et à la diffusion de documents scientifiques de niveau recherche, publiés ou non, émanant des établissements d'enseignement et de recherche français ou étrangers, des laboratoires publics ou privés. 


\title{
On One-Rule Grid Semi-Thue Systems
}

\author{
Michel Latteux \\ Laboratoire d'Informatique Fondamentale de Lille \\ Université Lille 1 \\ michel.latteux@lifl.fr \\ Yves Roos \\ Laboratoire d'Informatique Fondamentale de Lille \\ Université Lille 1 \\ yves.roos@lifl.fr
}

\begin{abstract}
The family of one-rule grid semi-Thue systems, introduced by Alfons Geser, is the family of one-rule semi-Thue systems such that there exists a letter $c$ that occurs as often in the left-hand side as the right-hand side of the rewriting rule. We prove that for any one-rule grid semi-Thue system $S$, the set $S(w)$ of all words obtainable from $w$ using repeatedly the rewriting rule of $S$ is a constructible context-free language. We also prove the regularity of the set $\operatorname{Loop}(S)$ of all words that start a loop in a one-rule grid semi-Thue systems $S$.
\end{abstract}

Keywords: one-rule semi-Thue system, termination, grid semi-Thue system.

\section{Introduction}

Semi-Thue systems, that are the non-symmetrical version of Thue systems introduced by Axel Thue in 1914, serve as a model for rewriting systems. Thus they are of primordial interest for computational problems. For several years they have been intensively studied and several deep results have been obtained [10, 3, 2, 21, 19, 22]. However some intriguing decidability problems remain open. With a semi-Thue system $S$, one associates the set $S_{\infty}$ of words that start an infinite derivation in $S$. The recursiveness of $S_{\infty}$ is called the termination problem for $S$ and the emptiness of $S_{\infty}$ is the uniform termination problem for $S$. The best result on the set $S_{\infty}$ is that the termination problem and the uniform termination problem are undecidable for 3-rules semi-Thue systems ([14]). Clearly the termination problem is decidable for length-preserving semi-Thue systems, contrarily to the uniform termination problem that has been shown undecidable for length-preserving semi-Thue systems 
([1]). This result remains true for 9-rules semi-Thue systems ([19]) and for length-two semi-Thue systems ([18]).

Another set naturally associated with semi-Thue systems is the set $\operatorname{Loop}(S)$ that brings together the words $w$ that start a non-null derivation in $S$ toward a word containing $w$ as a factor. For lengthpreserving semi-Thue systems, the emptiness of $\operatorname{Loop}(S)$ is equivalent to the emptiness of $S_{\infty}$ but this equivalence does not hold for arbitrary semi-Thue systems, as shown in [7] where a 2-rules semi-Thue system $S$ is presented with $\operatorname{Loop}(S)=\emptyset$ and $S_{\infty} \neq \emptyset$. Moreover, for an arbritary semi-Thue system $S$, the set $\operatorname{Loop}(S)$ need not be recursive.

One-rule semi-Thue systems are the simplest rewriting systems. Indeed, they are defined by two words $l, r$, noted $S=\{l \rightarrow r\}$. For a word $w, S(w)$ is the set of words obtainable from $w$ by replacing repeatedly $l$ by $r$. It is clear that, except the particular case $l=r, w \in S_{\infty}$ if and only if the set $S(w)$ is infinite. However, despite numerous efforts for more than twenty years, these decidability problems remain open and have become challenging problems. We observe that these problems can be explained in a few minutes to non-scientific people and surely they point out a deep lack of understanding of the rewriting notion. To get new ideas in order to solve these difficult problems, it seems natural to examine closely particular one-rule semi-Thue systems. Several interesting partial results have been obtained in that direction([20, 23, 16, 5, 6, 17]). Here we continue the study of a natural subclass of one-rule semi-Thue systems, introduced by Alfons Geser in [4], and called one-rule grid semi-Thue systems.

It is obvious that the system $S=\{l \rightarrow r\}$ with $l \neq r$ is uniformly terminating if $|r| \leq|l|$ or if there is a letter $x$ such that $|r|_{x}<|l|_{x}$. So, when studying the termination, we can assume that there is a letter $x$ such that $|r|_{x}>|l|_{x}$ and there is no letter $y$ such that $|r|_{y}<|l|_{y}$. Then a borderline case is when there is a unique letter $x$ such that $|r|_{x}>|l|_{x}$ and $|r|_{y}=|l|_{y}$ for $y \neq x$. The family of one-rule grid semi-Thue system satisfies a slightly more general condition. This family, that we denote $\mathcal{S}_{\text {grid }}$, is composed of all one-rule semi-Thue systems having a letter $c$ such that $|r|_{c}=|l|_{c}=k>00^{1}$. In [4], Alfons Geser has given a nice decidable characterization of one-rule grid semi-Thue systems that are uniformly terminating by proving that such a system is non-uniformly terminating if and only if it has a loop of length 1 or 2 . To know whether a one-rule semi-Thue system has a loop of length 1 or 2 was previously shown decidable by Winfried Kurth in [11].

First, we give a new formulation of this decidable characterization of non-uniformly terminating systems in $\mathcal{S}_{\text {grid }}$ : there exist words $x, y$ such that $l y$ is a left factor of $x r$ and $x l$ is a right factor of $r y$. We show that the words $x$ and $y$ are unique and give a simple way to compute these two words. This permits, when considering a nonterminating system $S=\{l \rightarrow r\} \in \mathcal{S}_{\text {grid }}$, to give a very precise form of the two words $l$ and $r$ and to get as a consequence that if $|r|_{c}=|l|_{c}=k$ is odd $l$ needs to be a factor of $r$.

Then we prove that, for any $S$ in $\mathcal{S}_{\text {grid }}$, the set $\operatorname{Loop}(S)$ is a constructible regular language by giving a simple rational expression. This property can not be generalized to arbitrary 1-rule semi-Thue system: for instance $\operatorname{Loop}(\{c \rightarrow c a c a\})$ is not regular. Concerning the link between $\operatorname{Loop}(S)$ and $S_{\infty}$, it is proved in [4] that the family $\mathcal{S}_{\text {grid }}$ satisfies the equality $S_{\infty}=S^{-1}\left(A^{*} \operatorname{Loop}(S) A^{*}\right)$, that is $w \in S_{\infty}$ if and only if there is a derivation from $w$ to a word in the regular language $A^{*} \operatorname{Loop}(S) A^{*}$ where $A$ is the alphabet of $l r$. Unfortunately, up to now, this relation does not imply that $S_{\infty}$ is a regular language as shown in [20] when $l \in a^{*} b^{*}$. Then we prove that for any $S$ in $\mathcal{S}_{\text {grid }}$ and for any

${ }^{1}$ In the original definition of Alfons Geser, a one-rule grid semi-Thue system is a one rule semi-Thue system $S=\{l \rightarrow r\}$ having a letter $c$ such that $|r|_{c} \leq|l|_{c}$. In this paper we do not consider the case $|r|_{c}<|l|_{c}$ for which $S$ is trivially uniformly terminating. 
word $w$, the set $S(w)$ is a constructible context-free language. Note that this result does not hold for arbitrary one-rule semi-Thue systems ([12]). As a matter of fact, $S(w)$ is a bounded context-free language. So we get both the decidability of the termination problem ${ }^{2}$ in $\mathcal{S}_{\text {grid }}$ and the decidability of the common descendant problem in $\mathcal{S}_{\text {grid }}$. In the last section, we give an argument in favour of the regularity of $S_{\infty}$ by proving that it is a regular language in the case $|r|_{c}=|l|_{c}=2$.

\section{Preliminaries and Notations}

In the following, $A$ will denote a finite alphabet, $A^{*}$ the free monoid over $A$ and $\varepsilon$ the empty word in $A^{*}$. For a word $w \in A^{*}$ and a letter $a \in A,|w|$ denotes the length of the word $w$ and $|w|_{a}$ denotes the number of occurrences of the letter $a$ in the word $w$.

Two words $u$ and $v$ are conjugate if there exist words $x$ and $y$ such that $u=x y$ and $v=y x$. It is well known that two words $u$ and $v$ are conjugate if and only if there exists a word $z$ such that $u z=z v$. A word $u$ is a factor of a word $v$ if there exist two words $w_{1}$ and $w_{2}$ such that $v=w_{1} u w_{2}$ and we denote by $\mathrm{F}(v)$ the set of the factors of the word $v$. We denote by $\operatorname{RF}(w)$ (respectively $\operatorname{LF}(w)$ ) the set of right factors (respectively left factors) of the word $w$, that is:

$$
\begin{aligned}
& \operatorname{RF}(w)=\left\{v \in A^{*} \mid \exists u \in A^{*}, w=u v\right\}, \\
& \operatorname{LF}(w)=\left\{u \in A^{*} \mid \exists v \in A^{*}, w=u v\right\} .
\end{aligned}
$$

A semi-Thue system over an alphabet $A$ is a subset $S \subseteq A^{*} \times A^{*}$. Members of $S$ are denoted $l \rightarrow r$ (or $l \rightarrow r$ if there is no ambiguity). One-step derivation, denoted $\vec{S}$ ( $\rightarrow$ if no ambiguity), is the binary relation over words defined by $: \forall u, v \in A^{*}, u \rightarrow v$ iff there exists $l \rightarrow r \in S$ and $\alpha, \beta \in A^{*}$ such that $u=\alpha l \beta$ and $v=\alpha r \beta$. The relation $\stackrel{*}{\rightarrow}($ resp. $\stackrel{+}{\rightarrow})$ is the reflexive and transitive closure (resp. transitive closure) of the relation $\rightarrow$ and, for any word $w \in A^{*}$, we shall denote $S(w)$ the set $S(w)=\left\{w^{\prime} \in A^{*} \mid w \underset{S}{\stackrel{*}{\rightarrow}} w^{\prime}\right\}$ and $S^{-1}(w)=\left\{w^{\prime} \in A^{*} \mid w^{\prime} \underset{S}{\stackrel{*}{\rightarrow}} w\right\}$. We extend these notations to languages: for any language $L \subseteq A^{*}, S(L)=\cup_{w \in L} S(w)$ and $S^{-1}(L)=\cup_{w \in L} S^{-1}(L)$. For a derivation $w=w_{0} \rightarrow w_{1} \cdots \rightarrow w_{n}=w^{\prime}, n$ is called the length of the derivation that will be denoted by $w \stackrel{n}{\rightarrow} w^{\prime}$.

We note $w \underset{S}{\stackrel{\infty}{\longrightarrow}}$ iff there is an infinite derivation starting on $w$ and we denote by $S_{\infty}$ the set $S_{\infty}=$ $\left\{w \in A^{*} \mid w \underset{S}{\stackrel{\infty}{\longrightarrow}}\right\}$.

For a semi-Thue system $S$, for any positive integer $n$, we denote $\operatorname{Loop}_{n}(S)=\left\{w \in A^{*} \mid \exists x, y \in\right.$ $\left.A^{*}, w \stackrel{n}{\rightarrow} x w y\right\}$ and $\operatorname{Loop}(S)=\cup_{n>0} \operatorname{Loop}_{n}(S)$.

The termination problem for the alphabet $A$ and the semi-Thue system $S \subseteq A^{*} \times A^{*}$ is the following:

instance: a word $w \in A^{*}$

\footnotetext{
${ }^{2}$ This result already appears implicitly inside proofs used by Alfons Geser in [4] to solve the uniform termination problem in $\mathcal{S}_{\text {grid. }}$
} 
question: Does every derivation (modulo $S$ ) starting on $w$ have finite length? (that is does $w \notin$ $S_{\infty}$ ?)

The uniform termination problem for a class $\mathcal{S}$ of semi-Thue systems is the following:

instance: an alphabet $A$ and a finite semi-Thue system $S \subseteq A^{*} \times A^{*}$ which belongs to $\mathcal{S}$

question: Do all derivations (modulo $S$ ) starting from all words $w \in A^{*}$ have finite length? (that is does $\left.S_{\infty}=\emptyset ?\right)$

To get shorter, we say that a system $S$ is nonterminating if the uniform termination problem has a negative answer for $S$. In the sequel, we focus on $\mathcal{S}_{\text {grid }}$, the family of one-rule grid semi-Thue systems.

Definition 2.1. A one-rule grid semi-Thue system $S=\{u \rightarrow v\}$ is a one-rule semi-Thue system such that there exists a letter $c$ with $|u|_{c}=|v|_{c}=k>0$.

\section{Uniform termination in $\mathcal{S}_{\text {grid }}$}

In this section, we state some consequences of the following Geser's result:

Proposition 3.1. ([4]) A one-rule grid semi-Thue system $S=\{u \rightarrow v\}$ is non-uniformly terminating iff one of the following properties is satisfied ${ }^{3}$ :

1. $u$ is a factor of $v$.

2. there exist words $g, h, k$ such that $u=g h, v=h k$ and $g g h$ is a factor of $h k k$.

Example 3.1. The simplest example of one-rule grid semi-Thue system satisfying the property 2 of proposition 3.1 but not the property 1 is $S=\{c a c \rightarrow a c c a\}$ with $g=c, h=a c, k=c a$ and $g g h=c c a c$ is a factor of $h k k=$ accaca.

Let $S=\{u \rightarrow v\} \in \mathcal{S}_{\text {grid }}$. The words $u$ and $v$ belong to $A^{*}$ where $A$ is an alphabet that contains a letter $c$ with $|u|_{c}=|v|_{c}=k>0$. If we denote $B=A \backslash\{c\}$, then

- $u=l c u_{1} \ldots u_{k-1} c r$

- $v=l^{\prime} c v_{1} \ldots v_{k-1} c r^{\prime}$

for some words $l, r, l^{\prime}, r^{\prime}, u_{1}, \ldots, u_{k-1}, v_{1}, \ldots, v_{k-1} \in B^{*}$. We get, as a consequence of the proposition 3.1, the following corollary that is also directly proved in [13]:

Corollary 3.1. Let $S=\{u \rightarrow v\} \in \mathcal{S}_{\text {grid }}$ with $u \neq v$. Then $S$ is nonterminating iff one of the two following properties is satisfied:

(i) $u$ is a factor of $v$

(ii) there exist $x, y, s, e \in A^{*}$ such that $x v=u y e$ and $v y=s x u$.

\footnotetext{
${ }^{3}$ More precisely, Alfons Geser proved in [4] that a one-rule grid semi-Thue system $S=\{u \rightarrow v\}$ is non-uniformly terminating if and only if $\operatorname{Loop}_{1}(S) \cup \operatorname{Loop}_{2}(S) \neq \emptyset$ that is proved decidable by Winfried Kurth in [11].
} 
Proof: Clearly, if (ii) is satisfied, $S$ is nonterminating since $x u \rightarrow x v=u y e \rightarrow$ vye $=$ sxue. Now, if $u \notin \mathrm{F}(v)$ and if the property 2 of the proposition 3.1 is satisfied, then $h k k=$ sgghe for some $s, e$. Since $|g|_{c}=|k|_{c}$, it follows $s, e \in B^{*}$. Suppose that $g, k \in B^{*}$ then, since $h \in l^{\prime} c A^{*}$ and $h k k=s g g h e$, we get $l^{\prime}=s g g l^{\prime}$ that implies $g=\varepsilon$ and $u=h \in \mathrm{F}(v)$, a contradiction. Thus $|g|_{c}=|k|_{c}>0$ and $k=y e$ for some $y \in A^{*}$. Taking $x=g$, we get $x v=g h k=u y e$, $v y e=v k=h k k=s g g h e=s x u e$, hence $v y=s x u$.

The following lemma states that if $u \neq v$ the conditions (i) and (ii) of the corollary 3.1 are mutually exclusive. This lemma also clarifies the relationship between the words $x, y, s$ and $e$ when the property (ii) of the corollary 3.1 is satisfied.

Lemma 3.1. Let $S=\{u \rightarrow v\} \in \mathcal{S}_{\text {grid }}$. If the property (ii) of the corollary 3.1 is satisfied and $u \neq v$, we have

(i) $|x|_{c}=|y|_{c}>0$ and $s, e \in B^{+}$,

(ii) $u \notin \mathrm{F}(v)$,

(iii) $u=x u^{\prime \prime}=u^{\prime} y$ where $u^{\prime \prime}$ is the longest word in $\operatorname{RF}(u) \cap \operatorname{LF}(v)$ and $u^{\prime}$ is the longest word in $\mathrm{LF}(u) \cap \mathrm{RF}(v)$

(iv) $x$ and $y$ are conjugate; $s$ and $e$ are conjugate.

Proof: If the property (ii) of the corollary 3.1 is satisfied, there are $e$ and $s$ in $A^{*}$ such that $x v=u y e$ (1) and $v y=s x u$ (2). From (1), we get $|x|_{c}=|y e|_{c}$ and from (2), $|y|_{c}=|s x|_{c}$. So $|x|_{c}=|y|_{c}$ and $|e|_{c}=|s|_{c}=0$ that is $e, s \in B^{*}$. Moreover $|x|_{c}=|y|_{c}>0$ otherwise $x$ and $y$ belong to $B^{*}$ and, from (1), we obtain $x l^{\prime}=l$ and from (2), $l^{\prime}=s x l$. It follows $x=s=\varepsilon$ and we similarly obtain $y=e=\varepsilon$. This leads to the contradiction $u=v$. Hence $x \in l c A^{*}$ and $y \in A^{*} c r$ so $v$ belongs to slc $A^{*} \cap A^{*}$ cre and $l^{\prime}=s l, r^{\prime}=r e$.

Let us now suppose that $u$ is a factor of $v$. We prove that this leads to a contradiction. Indeed, if $v=s^{\prime} u e^{\prime}$ for some $e^{\prime}, s^{\prime} \in B^{*}$, then the equality (1) gives $x s^{\prime} u e^{\prime}=u y e \in A^{*} c r e^{\prime} \cap A^{*}$ cre and it follows that $e^{\prime}=e$. Similarly equality (2) implies $s^{\prime}=s$ and we obtain $x s^{\prime} u=u y$ and $u e^{\prime} y=x u$. Therefore $e^{\prime}=s^{\prime}=\varepsilon$ and $u=v$; this contradiction proves (ii).

We can now finish the proof of (i): from (1) and (2), we get $x s x u=x v y=u y e y$ that implies $u \in$ $\operatorname{LF}\left((x s x)^{*}\right)$. Suppose $s=\varepsilon$ then $u \in \operatorname{LF}\left((x)^{*}\right)$ and $v y=x u \in \operatorname{LF}\left(x^{*}\right)$. It follows $v \in \operatorname{LF}\left(x^{*}\right)$ that implies $u \in \mathrm{F}(v)$, a contradiction. Similarly we can show $e \neq \varepsilon$.

For (iii), let us first observe that $|x|<|u|$ and $|y|<|u|$ : otherwise, we may assume that $|y| \leq|x|$; then $|x| \geq|u|$ and $x=u z$ for some word $z$. It follows that $x u=u z u \in \operatorname{RF}(v y)$ with $|y| \leq|x|=$ $|z u|$ and $u$ is a factor of $v$ which leads to a contradiction. Then we have $u=x u^{\prime \prime}=u^{\prime} y$ with $u^{\prime \prime}$ in $\operatorname{RF}(u) \cap \operatorname{LF}(v)$ and $u^{\prime}$ in $\operatorname{LF}(u) \cap \operatorname{RF}(v)$. We shall now prove that $u^{\prime \prime}$ is the longest word in $\operatorname{RF}(u) \cap \operatorname{LF}(v)$ and we could similarly show that $u^{\prime}$ is the longest word in $\operatorname{LF}(u) \cap \operatorname{RF}(v)$.

We have $x=l c u_{1} \ldots u_{j-1} c u_{j}^{\prime}$ with $u_{j}^{\prime} \in \operatorname{LF}\left(u_{j}\right)$ and, if we denote $p$ the first index such that $u_{p} \neq$ $v_{p}$ ( $p$ exists since $u \notin \mathrm{F}(v)$ ), we have $j \leq p$. Assume now that $j<p$ and set $z=l c u_{1} \ldots u_{p-j} c$, then we have $x s z \in \operatorname{LF}(u)$ and $s x z \in \operatorname{LF}(v)$. But this implies $s=\varepsilon$ and $u_{p}=u_{p-j}=v_{p}$, a contradiction. Thus $p=j$ and it follows $u_{j}=u_{j}^{\prime} s l$ and $v_{j}=u_{j}^{\prime} l$ with $s \neq \varepsilon$. Moreover, since $y e \in \operatorname{RF}(u e) \cap \operatorname{RF}(v)$ with $|x|_{c}=|y|_{c}$, we obtain $k \geq 2 p$ and $x s x^{\prime} \in \operatorname{LF}(u), s x x^{\prime} \in \operatorname{LF}(v)$, with $x^{\prime}=l c u_{1} \ldots u_{p-1} c$. 
In order to show that $u^{\prime \prime}$ is the longest word in $\operatorname{RF}(u) \cap \operatorname{LF}(v)$, assume that there exists some $z$ such that $|z|<|x|$ and $u \in \operatorname{LF}(z v)$. Then $|z|_{c}<|x|_{c}, z s x \in \operatorname{LF}\left(x s x^{\prime}\right)$ and sxs $x=$ szsxt with $t \neq \varepsilon$. Hence $x s$ is not a primitive word and $x s=\left(x_{r} s\right)^{q+1}$ where $x_{r} s$ is the primitive root of $x s$. That implies $x=\left(x_{r} s\right)^{q} x_{r}$ and $x^{\prime}=\left(x_{r} s\right) x_{r}^{\prime}$ with $x_{r}^{\prime} \in \operatorname{LF}\left(x_{r}\right),\left|x_{r}^{\prime}\right|_{c}=\left|x_{r}\right|_{c}>0, z=\left(x_{r} s\right)^{i} x_{r}$ with $i<q$ and $z s x=x z s$. Since $u \in \operatorname{LF}(z v)$, we have $x s x^{\prime} \in \operatorname{LF}\left(z s x x^{\prime}\right)=\operatorname{LF}\left(x s z x^{\prime}\right)$ and $x^{\prime}=\left(x_{r} s\right)^{q} x_{r}^{\prime} \in \operatorname{LF}\left(z x^{\prime}\right)=\operatorname{LF}\left(\left(x_{r} s\right)^{i} x_{r}\left(x_{r} s\right)^{q} x_{r}^{\prime}\right)$. Thus $\left(x_{r} s\right)^{q-i} x_{r}^{\prime} \in \operatorname{LF}\left(x_{r}\left(x_{r} s\right)^{q} x_{r}^{\prime}\right)$ and $s x_{r}^{\prime} \in \operatorname{LF}\left(x_{r} s\right)$. But that implies $s=\varepsilon$, a contradiction that finishes the proof of (iii). Observe that it implies the unicity of $x$ and $y$. Moreover, if there are several $c$ such that $|u|_{c}=|v|_{c}$, the value of $x$ and $y$ does not depend on the choice of a particular $c$.

Let us now prove (iv). Since $|x|_{c}=|y|_{c}$, we can write $x=x_{0} c x_{1} \ldots c x_{t}$ and $y=y_{0} c y_{1} \ldots c y_{t}$ with $\forall 0 \leq i \leq t, x_{i}, y_{i} \in B^{*}$. From (1) and (2), we can deduce $x v y=x s x u=$ uyey and it follows that $y e y$ and $x s x$ are conjugate. Thus $c y_{t} e y_{0} c \ldots c y_{t} y_{0} c \in \mathrm{F}\left((x s x)^{*}\right)$. Since $s, e \in B^{+}$, it is easy to verify that we must have $y_{t} e y_{0}=x_{t} s x_{0}$ and $y_{t} y_{0}=x_{t} x_{0}$. Suppose that $\left|y_{t}\right| \geq\left|x_{t}\right|$ (the case $\left|y_{t}\right| \leq\left|x_{t}\right|$ is symmetric) then $y_{t}=x_{t} z$ for some $z$ and it follows $z y_{0}=x_{0}$. Then we get $x_{t} z e y_{0}=x_{t} s z y_{0}$ so $s$ and $e$ are conjugate. Now from the equality $y_{t} e y y_{0}=x_{t} s x x_{0}$ we obtain $x_{t} z e y y_{0}=x_{t} s x z y_{0}$ that implies $z e y=s z y=s x z$ therefore $x$ and $y$ are conjugate.

Since the equalities $v y=s x u$ and $x v=u y e$ imply $x v y=x s x u=u y e y$, it follows that $u \in$ $\mathrm{LF}\left((x s x)^{*}\right), u \in \mathrm{RF}\left((y e y)^{*}\right), v \in \mathrm{LF}\left((s x x)^{*}\right)$ and $v \in \mathrm{RF}\left((y y e)^{*}\right)$. Then we can state the precise form of $u$ and $v$ for a nonterminating one-rule grid semi-Thue system:

Proposition 3.2. $S \in \mathcal{S}_{\text {grid }}$ is nonterminating iff one of the following three conditions is satisfied

1. $u \in \mathrm{F}(v)$

2. $u=(x s x)^{n} x^{\prime}$ and $v=(s x x)^{n} s x^{\prime}$ with $n>0$ and $x^{\prime} \in \operatorname{LF}(x) \cap \operatorname{LF}(s x)$

3. $u=(x s x)^{n} x s x^{\prime}$ and $v=(s x x)^{n} s x x^{\prime} e$ with $n \geq 0, x=x^{\prime} x^{\prime \prime}$ and $x^{\prime \prime} s=e x^{\prime \prime}$.

Proof: Let us first observe that these three conditions are sufficient by verifying that for any system $S$ satisfying 1,2 or $3, \operatorname{Loop}(S) \neq \emptyset$. It is clear for 1 since, in this case, $u \in \operatorname{Loop}_{1}(S)$. If $S$ satisfies 2 then $x(x s x)^{n} x^{\prime} \in \operatorname{Loop}_{2}(S)$ : indeed, in this case we get $x=x^{\prime} x^{\prime \prime}$ and $s x^{\prime}=x^{\prime} x^{\prime \prime \prime}$ for some words $x^{\prime \prime}, x^{\prime \prime \prime}$ and $x(x s x)^{n} x^{\prime} \rightarrow x(s x x)^{n} s x^{\prime}=(x s x)^{n} x^{\prime} x^{\prime \prime} s x^{\prime} \rightarrow(s x x)^{n} s x^{\prime} x^{\prime \prime} s x^{\prime}=$ $s x(x s x)^{n} x^{\prime} x^{\prime \prime \prime}$. Finally, if $S$ satisfies $3, x(x s x)^{n} x s x^{\prime} \rightarrow x(s x x)^{n} s x x^{\prime} e=(x s x)^{n} x s x^{\prime} x^{\prime \prime} x^{\prime} e \rightarrow$ $(s x x)^{n} s x x^{\prime} e x^{\prime \prime} x^{\prime} e=(s x x)^{n} s x x^{\prime} x^{\prime \prime} s x^{\prime} e=s x(x s x)^{n} x s x^{\prime} e$, therefore $x(x s x)^{n} x s x^{\prime} \in \operatorname{Loop}_{2}(S)$. It now remains to prove that the condition ( 1 or 2 or 3$)$ is necessary.

Suppose that 1 is not satisfied, then we can consider three cases since $u \in \operatorname{LF}\left((x s x)^{*}\right)$ :

(i) $u=(x s x)^{n} x^{\prime}$ with $x=x^{\prime} x^{\prime \prime}$.

First we can observe that $v=(s x x)^{n} s x^{\prime}$ since $v \in \operatorname{LF}\left((s x x)^{*}\right)$ and $|v|=|u|+|s|$. Moreover $n>0$ since $|x|_{c}<|u|_{c}$. It remains to prove that $x^{\prime} \in \mathrm{LF}(s x)$. The property (iii) of the lemma 3.1 gives $y \in \operatorname{RF}(u)$, therefore $y=x^{\prime \prime} x^{\prime}$ since $|x|=|y|$. From the equality $x v=$ uye we obtain $x(s x x)^{n} s x^{\prime}=(x s x)^{n} x^{\prime} x^{\prime \prime} x^{\prime} e$ and $x^{\prime} \in \operatorname{LF}\left(s x^{\prime}\right)$ since $|e|=|s|$.

(ii) $u=(x s x)^{n} x s^{\prime}$ with $s^{\prime} \in \operatorname{LF}(s)$.

Since $v \in \operatorname{LF}\left((s x x)^{*}\right)$ and $|v|=|u|+|s|$, it follows $v=(s x x)^{n} s x x^{\prime}$ with $\left|x^{\prime}\right|=\left|s^{\prime}\right|$. Then $x^{\prime} \in B^{*}$ since $|u|_{c}=|v|_{c}$ and $s \in B^{*}$. It follows that $x=x^{\prime} x^{\prime \prime}$ for some $x^{\prime \prime}$. Let us now consider the factorization $x=z c t$ with $t \in B^{*}$ (we know that $|x|_{c}>0$ ) then we obtain 
$u=u_{1} c t s^{\prime}$ and $v=v_{1} c t x^{\prime}$ with $\left|t s^{\prime}\right|=\left|t x^{\prime}\right|$ and $t s^{\prime}, t x^{\prime} \in B^{*}, u_{1}, v_{1} \in A^{*}$. This is a contradiction since $u=u_{1} c r$ and $v=v_{1}$ cre with $|r e|>|r|$. The case $u=(x s x)^{n} x s^{\prime}$ with $s^{\prime} \in \mathrm{LF}(s)$ is finally impossible.

(iii) $u=(x s x)^{n} x s x^{\prime}$ with $x^{\prime} \in \operatorname{LF}(x)$.

Since $v \in \operatorname{LF}\left((s x x)^{*}\right)$ and, from the property (iv) of the lemma 3.1, $|v|=|u|+|e|$, we get $v=(s x x)^{n} s x x^{\prime} x^{\prime \prime \prime}$ with $\left|x^{\prime \prime \prime}\right|=|e|$. Moreover $v \in \mathrm{RF}\left((y y e)^{*}\right)$ therefore $x^{\prime \prime \prime}=e$ and $v=(s x x)^{n} s x x^{\prime} e$. Now, from the equality $x v=u y e$, we can deduce that $y=x^{\prime \prime} x^{\prime}$ since $|x|=|y|$. Let us consider now the equality $v y=s x u$ : we get $(s x x)^{n} s x x^{\prime} e y=s x(x s x)^{n} x s x^{\prime}$ and it follows $x^{\prime} e y=x s x^{\prime}$. Since $x=x^{\prime} x^{\prime \prime}$ and $y=x^{\prime \prime} x^{\prime}$, we finally obtain $e x^{\prime \prime}=x^{\prime \prime} s$.

Example 3.2. Let $S=\left\{a^{2} c a^{5} c \rightarrow a^{4} c a^{3} c a^{2}\right\}$. In this case, $s=e=a^{2}$ and from the property (iii) of the lemma 3.1, $x=a^{2} c a$. Then we are in the case 3 of the proposition 3.2 with $n=0, x^{\prime}=a^{2} c$ and $x^{\prime \prime}=a$.

The condition 2 of the proposition 3.2 implies $x^{\prime} \in \operatorname{LF}\left(s x^{\prime}\right)$ so $x^{\prime} \in B^{*}$. It follows that $|u|_{c}=$ $2 n|x|_{c}$ is even. Similarly, the condition 3 of the proposition 3.2 implies that $x^{\prime \prime} \in B^{*}$. Then $\left|x^{\prime}\right|_{c}=|x|_{c}$ and $|u|_{c}=2 n|x|_{c}$ is even. We obtain:

Corollary 3.2. Let $S=\{u \rightarrow v\}$ such that $|u|_{c}=|v|_{c}$ is odd. Then $S$ is nonterminating iff $u \in \mathrm{F}(v)$.

\section{The construction of $\operatorname{Loop}(S)$ with $S$ in $\mathcal{S}_{\text {grid }}$}

The aim of this section is to prove that for any system $S=\{u \rightarrow v\}$ in $\mathcal{S}_{\text {grid }}$, the set $\operatorname{Loop}(S)$ is a constructible regular language. Clearly, if $u=v$ then $\operatorname{Loop}(S)=A^{*} u A^{*}$ and in the rest of this section, we suppose that $u \neq v$.

We shall use the following lemma:

Lemma 4.1. Let $S=\{u \rightarrow v\}$ in $\mathcal{S}_{\text {grid }}$ that is nonterminating with $u \notin \mathrm{F}(v)$. If $z_{0} c w_{0} c z_{0}^{\prime} \stackrel{4}{\rightarrow}$ $z_{4} c w_{4} c z_{4}^{\prime}$ with $z_{0}, z_{0}^{\prime}, z_{4}, z_{4}^{\prime} \in B^{*}$ and $\left|w_{0}\right|=\left|w_{4}\right|$ then $z_{0} c w_{0} c z_{0}^{\prime} \in B^{*}(x u+u y) B^{*}$.

Proof: We consider the derivation $z_{0} c w_{0} c z_{0}^{\prime} \rightarrow z_{1} c w_{1} c z_{1}^{\prime} \rightarrow z_{2} c w_{2} c z_{2}^{\prime} \rightarrow z_{3} c w_{3} c z_{3}^{\prime} \rightarrow z_{4} c w_{4} c z_{4}^{\prime}$ with $z_{i}, z_{i}^{\prime} \in B^{*}$. Observe first that $\left|c w_{0} c\right|_{c}>|u|_{c}$ otherwise we can not apply a derivation step on $z_{1} c w_{1} c z_{1}^{\prime}$ since $u \notin \mathrm{F}(v)$. That implies that the first and the last occurrences of the letter $c$ can not be both involved in a same step of the derivation. From the proposition 3.2, $\left|w_{i+1}\right|=\left|w_{i}\right|$ if the derivation involves the first or the last occurrence of the letter $c$, else $\left|w_{i+1}\right|>\left|w_{i}\right|$. From the hypothesis, we are in the first case and $\forall i,\left|w_{i}\right|=\left|w_{0}\right|$. We can also suppose that $z_{0}=l$ and $z_{0}^{\prime}=r$. Then $u \in \operatorname{LF}\left(l c w_{0} c r\right) \cup \operatorname{RF}\left(l c w_{0} c r\right)$ and we consider the case $l c w_{0} c r=u y_{0} \rightarrow v y_{0}$. Since $s u \notin \mathrm{LF}(v)$, we necessarily have $u \in \operatorname{RF}\left(v y_{0}\right)$. It follows that $v y_{0}=s x_{1} u \rightarrow s x_{1} v=s u y_{1} e \rightarrow$ $s v y_{1} e=s^{2} x_{2} u e$. We can verify that $\left|y_{0}\right|=\left|y_{1}\right|$ and $y_{0}, y_{1} \in \operatorname{RF}(u)$. Finally $y_{0}=y_{1}$ and we get $v y_{0}=s x_{1} u$ and $x_{1} v=u y_{0} e$. Now, from the property (ii) of the corollary 3.1 and from the property (iii) of the lemma 3.1, we get $y_{0}=y, x_{1}=x$ that imply $l c w_{0} c r=u y$. Observe that if $z_{0} \neq l$ or $z_{0}^{\prime} \neq r$ we still have $z_{0} c w_{0} c z_{0}^{\prime} \in B^{*} u y B^{*}$. If $u \in \operatorname{RF}\left(l c w_{0} c r\right)$, then $z_{0} c w_{0} c z_{0}^{\prime} \in B^{*} x u B^{*}$ that proves the lemma. 
Let us denote, for any word $w \in A^{*} c A^{*}, \operatorname{int}(w)=\left(B^{*}\right)^{-1} w\left(B^{*}\right)^{-1} \cap c A^{*} \cap A^{*} c$.

Lemma 4.2. Let $S$ be nonterminating with $u \neq v$.

1. If $w \stackrel{*}{\rightarrow} w^{\prime}$ with $u \in \mathrm{F}(v)$ or $|w|_{c}>|u|_{c}$, then $\left|\operatorname{int}\left(w^{\prime}\right)\right| \geq|\operatorname{int}(w)|$,

2. if $w \stackrel{+}{\rightarrow} w^{\prime} \stackrel{*}{\rightarrow} z w z^{\prime}$ then $|\operatorname{int}(w)|=\left|\operatorname{int}\left(w^{\prime}\right)\right|$

Proof: For the property 1, it is sufficient to prove that $w \rightarrow w^{\prime} \operatorname{implies}\left|\operatorname{int}\left(w^{\prime}\right)\right| \geq|\operatorname{int}(w)|$. That is clear if $u \in \mathrm{F}(v)$ since $|v|>|u|$ and $\operatorname{int}(v)=\operatorname{int}(u)$. If $u \notin \mathrm{F}(v)$ then the property (ii) of the corollary 3.1 is satisfied. Since $|w|_{c}>|u|_{c}$ we have to consider three cases:

1. $w=z u w^{\prime \prime}$ and $w^{\prime}=z v w^{\prime \prime}$ with $z \in B^{*}, w^{\prime \prime} \in\left(A^{*} \backslash B^{*}\right)$,

2. $w=w^{\prime \prime} u z$ and $w^{\prime}=w^{\prime \prime} v z$ with $z \in B^{*}, w^{\prime \prime} \in\left(A^{*} \backslash B^{*}\right)$,

3. $w=w^{\prime \prime} u w^{\prime \prime \prime}$ with $w^{\prime \prime}, w^{\prime \prime \prime} \in\left(A^{*} \backslash B^{*}\right)$.

Let us consider the case 1. From the property (iv) of the lemma 3.1, $|\operatorname{int}(u)|=|\operatorname{int}(v)|+|e|$, moreover $u \in A^{*} c r$ and $v \in A^{*}$ cre so we get $\left|\operatorname{int}\left(w^{\prime}\right)\right|=|\operatorname{int}(w)|$. The second case can be proved similarly and for the third case, we clearly obtain $\left|\operatorname{int}\left(w^{\prime}\right)\right|>|\operatorname{int}(w)|$ since $|u|<|v|$. For the property 2 , we have $\operatorname{int}\left(z w z^{\prime}\right)=\operatorname{int}(w)$ and $w \in S_{\infty}$. So, if $u \notin \mathrm{F}(v),|w|_{c}>|u|_{c}$ from the lemma 4.1. Then property 1 yields: $|\operatorname{int}(w)| \leq\left|\operatorname{int}\left(w^{\prime}\right)\right| \leq\left|\operatorname{int}\left(z w z^{\prime}\right)\right|=|\operatorname{int}(w)|$.

Lemma 4.3. If the property (ii) of the corollary 3.1 is satisfied and $u \neq v$ then
1. $v \notin \mathrm{F}\left(x u B^{*}\right)$,
2. $v \notin \mathrm{F}\left(B^{*} u y\right)$,
3. $x u \notin A^{*} u A^{+}$,
4. $u y \notin A^{+} u A^{*}$.

Proof: From the property (iii) of the lemma 3.1, $u=g y$ where $g$ is the longest word in $\operatorname{LF}(u) \cap$ $\operatorname{RF}(v)$. Assume first that $v \in \mathrm{F}(x u)$. Since $v \in s l c A^{*}$ and $x u \in l c A^{*}, v \notin \mathrm{LF}(x u)$. Then $u=g^{\prime} y^{\prime}$ with $g^{\prime} \in \operatorname{LF}(u) \cap \operatorname{RF}(v)$ and $\left|y^{\prime}\right|<|x|=|y|$. Thus $|g|<\left|g^{\prime}\right|$, a contradiction. Assume now that $v \in \mathrm{F}\left(x u B^{*}\right)$. Since $v \in A^{*} c r e$ and $u \in A^{*} c r$, we get $v \in \mathrm{RF}(x u e)$ and $v=u e$, a contradiction. Assume now that $x u \in A^{*} u A^{+}$. Consider the equality $v y=s x u$. If $u \in \operatorname{LF}(x u)$ then $v=s u$, a contradiction. Otherwise, $u=g^{\prime} y^{\prime}$ with $g^{\prime} \in \operatorname{LF}(u) \cap \operatorname{RF}(v)$ and $\left|y^{\prime}\right|<|y|$. Thus $|g|<\left|g^{\prime}\right|$, a contradiction. Properties 2 and 4 can be proved similarly.

We obtain as a consequence:

Corollary 4.1. If the property (ii) of the corollary 3.1 is satisfied and $u \neq v$ then $S^{-1}\left(B^{*}(x u+\right.$ uy) $\left.B^{*}\right)=B^{*}(x u+u y) B^{*}$. 
Proof: Let us prove that $S^{-1}\left(B^{*} x u B^{*}\right) \subseteq B^{*}(x u+u y) B^{*}$. It is clearly sufficient to consider a single step of derivation. Let $w_{1} u w_{2} \rightarrow w_{1} v w_{2}=w_{1}^{\prime} x u w_{2}^{\prime}$ with $w_{1}, w_{2} \in A^{*}$ and $w_{1}^{\prime}, w_{2}^{\prime} \in B^{*}$. From the lemma 4.3, $v \notin \mathrm{F}\left(x u B^{*}\right)$ therefore there exist $t \in B^{*}$ and $z \in A^{*}$ such that $w_{1}^{\prime}=w_{1} t$, $w_{2}=z w_{2}^{\prime}$ and $t x u=v z$. Since $v \in \operatorname{slc} A^{*}$ and $x \in l c A^{*}$ it follows $t=s$ that implies $z=y$ and $w_{1} u w_{2}=w_{1} u y w_{2}^{\prime} \in B^{*} u y B^{*}$. We can prove similarly that $S^{-1}\left(B^{*} u y B^{*}\right) \subseteq B^{*}(x u+u y) B^{*}$.

\section{Proposition 4.1.}

1. If $v=s u$ with $s \in B^{+}$then $\operatorname{Loop}(S)=\operatorname{RF}\left(s^{*}\right) u A^{*}$,

2. If $v=u e$ with $e \in B^{+}$then $\operatorname{Loop}(S)=A^{*} u \operatorname{LF}\left(e^{*}\right)$,

3. If $v=$ sue with $s, e \in B^{+}$then $\operatorname{Loop}(S)=\operatorname{RF}\left(s^{*}\right) u \operatorname{LF}\left(e^{*}\right)$.

Proof: Let $S=\{u \rightarrow s u\}$ with $s \in B^{+}$and $w \in \operatorname{Loop}(S)$. Then $w=\alpha u \beta \rightarrow \alpha s u \beta \stackrel{*}{\rightarrow} z \alpha u \beta z^{\prime}$ with $z, z^{\prime} \in B^{*}$. From the lemma 4.2, we have $|\operatorname{int}(\alpha s u \beta)|=|\operatorname{int}(\alpha u \beta)|$ and, since $s \in B^{+}$, we get $\alpha \in B^{*}$. Moreover we obtain by induction on the length $n>0$ of the derivation that $z \alpha u \beta z^{\prime}=\alpha s^{n} u \beta$. It follows $\alpha s^{n}=z \alpha$ and $\alpha \in \operatorname{RF}\left(\alpha s^{i \times n}\right)$ for any integer $i>0$. Therefore $\alpha \in \operatorname{RF}\left(s^{*}\right)$ and $\operatorname{Loop}(S) \subseteq \operatorname{RF}\left(s^{*}\right) u A^{*}$. Since the converse inclusion is immediate we finally get $\operatorname{Loop}(S)=\operatorname{RF}\left(s^{*}\right) u A^{*}$. The other cases can be proved similarly.

Lemma 4.4. If the property (ii) of the corollary 3.1 is satisfied and $u \neq v$ then for any word $\alpha, \beta \in$ $B^{*}$

1. $S(\alpha x u \beta)=\left\{\alpha s^{n} x u e^{n} \beta \mid n \geq 0\right\} \cup\left\{\alpha s^{n-1} u y e^{n} \beta \mid n>0\right\}$,

2. $S(\alpha u y \beta)=\left\{\alpha s^{n} u y e^{n} \beta \mid n \geq 0\right\} \cup\left\{\alpha s^{n-1} x u e^{n} \beta \mid n>0\right\}$.

Proof: We prove that $S(\alpha x u \beta)=\left\{\alpha s^{n} x u e^{n} \beta \mid n \geq 0\right\} \cup\left\{\alpha s^{n-1} u y e^{n} \beta \mid n>0\right\}$ : from the lemma 4.3, $x u \notin A^{*} u A^{+}$and $u y \notin A^{+} u A^{*}$ therefore the first steps of a derivation from $w$ are $w=\alpha x u \beta \rightarrow \alpha x v \beta=\alpha u y e \beta \rightarrow \alpha v y e \beta=\alpha$ sxue $\beta$ and the property is proved by induction on the length of the derivation. The second property of this lemma can be proved similarly.

Proposition 4.2. If the property (ii) of the corollary 3.1 is satisfied and $u \neq v$ then

$$
\operatorname{Loop}(S)=\operatorname{RF}\left(s^{*}\right)(x u+u y) \operatorname{LF}\left(e^{*}\right)
$$

Proof: Let $w \in \operatorname{Loop}(S)$ then $w \stackrel{n}{\rightarrow} z w z^{\prime}$ with $z, z^{\prime} \in B^{*}$ and $n>0$. We can suppose $n \geq 4$ and there exists a derivation $w \rightarrow w_{1} \rightarrow w_{2} \rightarrow w_{3} \rightarrow w_{4} \stackrel{*}{\rightarrow} z w z^{\prime}$ with $z, z^{\prime} \in B^{*}$. From the lemma 4.2, we get $\left|\operatorname{int}\left(w_{4}\right)\right|=|\operatorname{int}(w)|$ and, from the lemma 4.1, we get $w=\alpha x u \beta$ or $w=$ $\alpha u y \beta$ with $\alpha, \beta \in B^{*}$. Let us suppose that $w=\alpha x u \beta$ then $S(w)=\left\{\alpha s^{n} x u e^{n} \beta \mid n \geq 0\right\} \cup$ $\left\{\alpha s^{n-1}\right.$ uye $\left.\beta \mid n>0\right\}$ from the lemma 4.4. Moreover, from the lemma 4.3 we deduce that $x u \neq u y$ since $u \notin \operatorname{LF}(x u)$. On the other hand, from the property (iii) of the lemma 3.1, we have $x u \in l c A^{*} c r$ and $u y \in l c A^{*} c r$ then it follows that $\operatorname{int}(u y) \neq \operatorname{int}(x u)=\operatorname{int}(w)$. That implies that if $w=\alpha x u \beta \stackrel{+}{\rightarrow} z w z^{\prime}=z \alpha x u \beta z^{\prime}$ we must have $z \alpha x u \beta z^{\prime}=\alpha s^{n} x u e^{n} \beta$ for some $n>0$ therefore $\alpha \in \operatorname{RF}\left(s^{*}\right)$ and $\beta \in \operatorname{LF}\left(e^{*}\right)$. The case $w=\alpha u y \beta$ is symmetric and we finally get $\operatorname{Loop}(S) \subseteq \operatorname{RF}\left(s^{*}\right)(x u+u y) \operatorname{LF}\left(e^{*}\right)$. We can easily verify the converse inclusion since $x u \stackrel{*}{\rightarrow} s x u e$ and $u y \stackrel{*}{\rightarrow}$ suye. 
We directly obtain from the two previous propositions:

Corollary 4.2. If $S \in \mathcal{S}_{\text {grid }}$ then $\operatorname{Loop}(S)$ is a constructible regular language.

The condition $S \in \mathcal{S}_{\text {grid }}$ in the corollary 4.2 is necessary as shown by the following example:

Example 4.1. Consider $S=\{c \rightarrow c a c a\}$. We shall show that $\operatorname{Loop}(S) \cap c(c a)^{+} a^{*}=L=$ $c\left\{(c a)^{n} a^{p} \mid n>p \geq 0\right\}$. Since $c \stackrel{*}{\rightarrow}(c a)^{n-1} c a^{n-1}$, it follows that for $n>p \geq 0, c(c a)^{n} a^{p} \stackrel{*}{\rightarrow}$ $c(c a)^{n} a^{p} a^{n-1-p}(c a)^{n-1} a^{p}$ and $L \subseteq \operatorname{Loop}(S)$. For the reverse inclusion, remark that $(c a+a)^{*}$ is $S$ closed. So, if $c w=c(c a)^{n} a^{p} \stackrel{*}{\rightarrow} \alpha c w \beta$ for some $\alpha, \beta \in A^{*}$, then $\alpha=\varepsilon$ and $w \stackrel{+}{\rightarrow} w \beta$ with $|\beta|_{c}>$ 0 . Thus $\beta=\beta^{\prime} a^{p},(c a)^{n} \stackrel{*}{\rightarrow}(c a)^{n} a^{p} \beta^{\prime} \in \operatorname{LF}(S(c))$ and $p<n$ since $\forall z \in \operatorname{LF}(S(c)), 2|z|_{c}>|z|_{a}$.

\section{5. $\quad$ Testing membership in $S_{\infty}$ with $S$ in $\mathcal{S}_{\text {grid }}$}

The aim of this section is to show that the termination problem of a system $S \in \mathcal{S}_{\text {grid }}$ is decidable. Recall that this problem is, given $S \in \mathcal{S}_{\text {grid }}$ and a word $w \in A^{*}$ to know whether $w \in S_{\infty}$. Clearly, for a system $S=\{u \rightarrow v\}$ with $u \in \mathrm{F}(v), S_{\infty}=A^{*} u A^{*}$ and we conjecture that for any system $S \in \mathcal{S}_{\text {grid }}$ the set $S_{\infty}$ is a constructible regular language. This is true when $|u|_{c}=|v|_{c}=2$ as it is proved in the next section but here we must find another way in order to prove that $S_{\infty}$ is a recursive language for any $S \in \mathcal{S}_{\text {grid }}$. More precisely, we show that for any $S \in \mathcal{S}_{\text {grid }}$ and any word $w$, the set $S(w)$ is a constructible bounded context-free language.

Definition 5.1. ([9]) A language $L \subseteq A^{*}$ is said to be bounded if there exist words $w_{1}, \ldots, w_{n} \in A^{*}$ such that $L \subseteq w_{1}^{*} \ldots w_{n}^{*}$.

As a matter of fact, it would be sufficient to prove that for any $S \in \mathcal{S}_{\text {grid }}$ and any word $w$, the set $S(w)$ is a constructible context-free language in order to decide whether $S(w)$ is finite. Nevertheless bounded languages have nice structural properties that will also permit to solve in this section the common descendant problem for any $S \in \mathcal{S}_{\text {grid }}$ that is to decide, given two words $w$ and $w^{\prime}$, whether $S(w) \cap S\left(w^{\prime}\right)$ is not empty. We also observe that the image $S(w)$ of a word $w$ by an arbitrary one-rule semi-Thue system $S$ need not be context-free([12]).

Following Alfons Geser in [4], we first establish that if a derivation is applied to a word long enough, this word can then be factorized so that the derivation applies independently on each factor of the word. Let us denote $N^{\prime}=\max \left\{\left|c u_{i}\right| \mid i<k\right\}$ and $N=\max \left\{N^{\prime},|l|,|r|\right\}$.

Lemma 5.1. Let $w=z^{\prime} c m^{\prime} m^{\prime \prime} c z^{\prime \prime}$ with $m^{\prime}, m^{\prime \prime} \in B^{*}$ and $z^{\prime}, z^{\prime \prime} \in A^{*}$. If $\left|m^{\prime}\right| \geq N$ and $\left|m^{\prime \prime}\right| \geq$ $N$ then $S(w)=S\left(z^{\prime} c m^{\prime}\right) S\left(m^{\prime \prime} c z^{\prime \prime}\right)$.

Proof: Clearly $S\left(z^{\prime} c m^{\prime}\right) S\left(m^{\prime \prime} c z^{\prime \prime}\right) \subseteq S(w)$. Conversely, let $w^{\prime} \in S(w)$, we prove by induction on the length $n$ of the derivation from $w$ to $w^{\prime}$ that $w^{\prime} \in S\left(z^{\prime} c m^{\prime}\right) S\left(m^{\prime \prime} c z^{\prime \prime}\right)$. The base case $w=w^{\prime}$ is immediate. Otherwise $w \rightarrow w^{\prime \prime} \stackrel{n-1}{\longrightarrow} w^{\prime}$. Since $\left|m^{\prime} m^{\prime \prime}\right|>N$, the first step of the derivation applies on an occurrence of $u$ that can only appear in $z^{\prime} \mathrm{cm}^{\prime}$ or in $m^{\prime \prime} c z^{\prime \prime}$. Suppose it is in $z^{\prime} \mathrm{cm}^{\prime}$, then $z^{\prime} c m^{\prime} \rightarrow z_{1}^{\prime} c m_{1}^{\prime}$ with $m_{1}^{\prime} \in B^{*},\left|m_{1}^{\prime}\right| \geq\left|m^{\prime}\right|$ and $w^{\prime \prime}=z_{1}^{\prime} c m_{1}^{\prime} m^{\prime \prime} c z^{\prime \prime}$. Now, we can apply the induction hypothesis on $w^{\prime \prime} \stackrel{n-1}{\longrightarrow} w^{\prime}: w^{\prime} \in S\left(z_{1}^{\prime} c m_{1}^{\prime}\right) S\left(m^{\prime \prime} c z^{\prime \prime}\right) \subseteq S\left(z^{\prime} c m^{\prime}\right) S\left(m^{\prime \prime} c z^{\prime \prime}\right)$. The other case can be proved similarly. 
We directly deduce:

Lemma 5.2. Let $z c w c z^{\prime}$ with $z, z^{\prime} \in B^{*}$ and $w \in A^{*}$. If $|c w c| \geq 2 \times\left(|w|_{c}+2\right) \times N$ then there exist two constructible words $w^{\prime}, w^{\prime \prime} \in\left(A^{*} \backslash B^{*}\right)$ such that $z c w c z^{\prime}=w^{\prime} w^{\prime \prime}$ and $S\left(z c w c z^{\prime}\right)=$ $S\left(w^{\prime}\right) S\left(w^{\prime \prime}\right)$.

Then we can state:

Proposition 5.1. Let $S \in \mathcal{S}_{\text {grid }}$ satisfying the property (ii) of the corollary 3.1.

1. For any word $w \in A^{*}$, the set $S(w)$ is a bounded context-free language.

2. $S_{\infty}=S^{-1}\left(A^{*}(x u+u y) A^{*}\right)=S^{-1}\left(A^{*} \operatorname{Loop}(S) A^{*}\right)$.

Proof: The proof is based on an induction over $|w|_{c}$. For the property 2, we have only to prove $S_{\infty} \subseteq S^{-1}\left(A^{*}(x u+u y) A^{*}\right)$ since the inclusions $S^{-1}\left(A^{*}(x u+u y) A^{*}\right) \subseteq S^{-1}\left(A^{*} \operatorname{Loop}(S) A^{*}\right) \subseteq$ $S_{\infty}$ are clear.

- If $|w|_{c}<|x u|_{c}$ then $S(w)$ is finite (therefore bounded context-free) from the lemma 4.1,

- If $|w|_{c}=|x u|_{c}$ then

- if $w \notin B^{*}(x u+u y) B^{*}$ then $w \notin S^{-1}\left(B^{*}(x u+u y) B^{*}\right)$ from the corollary 4.1 and it follows that $S(w)$ is finite from the lemma 4.1,

- if $w \in B^{*}(x u+u y) B^{*}$ then, from the lemma 4.4, $S(w)$ is a (constructible) bounded context-free language and $w$ is in $S^{-1}\left(A^{*}(x u+u y) A^{*}\right)$

- If $|w|_{c}>|x u|_{c}$, we make a new induction on $K_{w}=2 \times\left(|w|_{c}\right) \times N-|\operatorname{int}(w)|$. If $K_{w} \leq 0$ then it follows from the lemma 5.2 that there exist two constructible words $w^{\prime}, w^{\prime \prime} \in\left(A^{*} \backslash B^{*}\right)$ such that $w=w^{\prime} w^{\prime \prime}$ and $S(w)=S\left(w^{\prime}\right) S\left(w^{\prime \prime}\right)$. As $\left|w^{\prime}\right|_{c}<|w|_{c}$ and $\left|w^{\prime \prime}\right|_{c}<|w|_{c}$, we can apply the induction hypothesis and it follows that $S(w)$ is a constructible bounded context-free language. If $K_{w}>0$, let us denote $S_{1}=\left\{w^{\prime} \in A^{*} \mid w \stackrel{<4}{\longrightarrow} w^{\prime}\right\}$ and $S_{2}=\left\{w^{\prime} \in A^{*} \mid w \stackrel{4}{\rightarrow}\right.$ $\left.w^{\prime}\right\}$. Clearly $S(w)=S_{1} \cup\left(\bigcup_{w^{\prime} \in S_{2}} S\left(w^{\prime}\right)\right)$. The family of bounded languages is closed by finite union, consequently it remains to prove that for any word $w^{\prime}$ in $S_{2}, S\left(w^{\prime}\right)$ is a bounded context-free language. Since $w \notin B^{*}(x u+u y) B^{*}$ it follows from the lemma 4.1 and the lemma 4.2 that, for any word $w^{\prime} \in S_{2}$, $\left|\operatorname{int}\left(w^{\prime}\right)\right|>|\operatorname{int}(w)|$. Then $K_{w^{\prime}}<K_{w}$ and, by the induction hypothesis, $S\left(w^{\prime}\right)$ is a bounded context-free language, moreover if $w^{\prime} \in S_{\infty}$ then $w^{\prime} \in S^{-1}\left(A^{*}(x u+u y) A^{*}\right)$ and it follows that $w \in S^{-1}\left(A^{*}(x u+u y) A^{*}\right)$ which concludes the proof of the proposition.

When $u \in \mathrm{F}(v)$, a similar proof can be used to show that the property 1 is also satisfied in this case. Then we get as a corollary:

Corollary 5.1. The termination problem is decidable for any system $S \in \mathcal{S}_{\text {grid }}$.

Since it is proved in [8] that the non-emptiness of the intersection of two bounded context-free languages is decidable, we also obtain:

Corollary 5.2. The common descendant problem is decidable for any system $S \in \mathcal{S}_{\text {grid }}$. 


\section{The special case $|u|_{c}=|v|_{c}=2$}

It has been shown in the section 4 that $\operatorname{Loop}(S)$ is regular for $S$ in $\mathcal{S}_{\text {grid }}$ and that this result does not hold for arbitrary one-rule semi-Thue system. The status of $S_{\infty}$ is different. Géraud Sénizergues has proved in [20] that $S_{\infty}$ is regular when $u \in a^{*} b^{*}$, but the regularity of $S_{\infty}$ is an open problem for arbitrary one-rule semi-Thue system. Note that the regularity of $S_{\infty}$ does not hold for two-rules grid semi-Thue system as shown by the example $S=\{a c a \rightarrow c, c c \rightarrow c c a\}$ since it is easy to see that $S_{\infty}=A^{*} c A^{*} c A^{*} c A^{*} \cup\left\{a^{i} c a^{p} c a^{q} \mid p \leq i+q\right\}$. We conjecture that $S_{\infty}$ is a constructible regular language for any $S$ in $\mathcal{S}_{\text {grid }}$. As we are unable to prove this fact, we give an argument in favour by proving it in the simpler case $|u|_{c}=|v|_{c}=2$. The following proof shows also that the structure of $S_{\infty}$ can be involved and that the proof in the general case $S$ in $\mathcal{S}_{\text {grid }}$ could be tricky.

Clearly the question arises only when $S$ satisfies the property (ii) of the corollary 3.1 since, when $u \in \mathrm{F}(v), S_{\infty}=A^{*} u A^{*}$. Then we consider in the following a nonterminating system $S=\{u \rightarrow v\}$ with $u=l c u_{1} c r, v=s l c v_{1} c r e, x v=u y e$ and $v y=s x u$ for $u_{1}, v_{1}, s, e \in B^{*}$ and $x, y \in A^{*}$. Then there exist words $u_{1}^{\prime}$ and $u_{1}^{\prime \prime}$ such that $u_{1}=u_{1}^{\prime} s l=r e u_{1}^{\prime \prime}, v_{1}=u_{1}^{\prime} l=r u_{1}^{\prime \prime}, x=l c u_{1}^{\prime}$ and $y=u_{1}^{\prime \prime} c r$

Let us denote $S_{\infty}^{\text {min }}=S_{\infty} \backslash\left(A S_{\infty} \cup S_{\infty} A\right)$ and, for any word $w \in A^{*}, L_{w}=S^{-1}\left(A^{*} w\right) \backslash\left(S_{\infty} \cup\right.$ $\left.A S^{-1}\left(A^{*} w\right)\right)$ and $R_{w}=S^{-1}\left(w A^{*}\right) \backslash\left(S_{\infty} \cup S^{-1}\left(w A^{*}\right) A\right)$.

Lemma 6.1. $S_{\infty}^{\min } \subseteq L_{x l} c u_{1} c R_{r} \cup L_{l} c u_{1} c R_{r y}$.

Proof: Let us consider $w_{0} \in S_{\infty}^{\min }$ and let $n$ be the length of the shortest derivation from $w_{0}$ to any word in $A^{*}(x u+u y) A^{*}$. Let us suppose that there exists a derivation from $w_{0}$ to $w_{n} \in A^{*}(x u) A^{*}$. Then $w_{0} \stackrel{*}{\rightarrow} w_{1} \stackrel{*}{\rightarrow} w_{i} \stackrel{*}{\rightarrow} w_{n}$ and $w_{n}=p_{n} c q_{n} c r_{n}$ with $p_{n} \in A^{*} x l, q_{n}=u_{1}$ and $r_{n} \in r A^{*}$. For any $i \in[0, n]$, let us consider the factorization $w_{i}=p_{i} c q_{i} c r_{i}$ with $\left|p_{i}\right|_{c}=\left|p_{n}\right|_{c}$ and $q_{i} \in B^{*}$. We claim that $q_{0}=u_{1}$, else let $j$ be the greatest index such that $q_{j} \neq u_{1}$. Then $w_{j+1}=p_{j+1} c u_{1} c r_{j+1}$ and $p_{j+1} \stackrel{*}{\rightarrow} p_{n}, r_{j+1} \stackrel{*}{\rightarrow} r_{n}$. This will lead to the following contradiction: there exists a derivation from $w_{0}$ to a word in $A^{*}(x u+u y) A^{*}$ whose length is strictly less than $n$. It follows that for any $i, q_{i}=u_{1}$ and $p_{0} \stackrel{*}{\rightarrow} p_{n}, r_{0} \stackrel{*}{\rightarrow} r_{n}$ therefore $p_{0} \in S^{-1}\left(A^{*} x l\right), r_{0} \in S^{-1}\left(r A^{*}\right)$. Since $w_{0} \in S_{\infty}^{\min }$, it follows $p_{0} \in L_{x l}, r_{0} \in R_{r}$ and $w_{0} \in L_{x l} c u_{1} c R_{r}$. Let us distinguish two cases for the step $w_{j} \rightarrow w_{j+1}$ :

1. $q_{j}=u_{1}^{\prime} l, r_{j}=u_{1}$ cr $z$ and $r_{j+1}=v_{1}$ crez.

Then $p_{n} c u_{1}^{\prime} \in A^{*} l c u_{1}^{\prime}=A^{*} x$ and $w^{\prime}=p_{n} c q_{j} c r_{j}=p_{n} c u_{1}^{\prime} l c u_{1} c r z \in A^{*} x u A^{*}$. Clearly, the length of the derivation $w_{0} \stackrel{*}{\rightarrow} w_{j}=p_{j} c q_{j} c r_{j} \stackrel{*}{\rightarrow} w^{\prime}=p_{n} c q_{j} c r_{j}$ is strictly smaller than $n$.

2. $q_{j}=r u_{1}^{\prime \prime}, p_{j}=z l c u_{1}$ and $p_{j+1}=z s l c v_{1}$.

Then $u_{1}^{\prime \prime} c r_{n} \in u_{1}^{\prime \prime} c r A^{*}=y A^{*}$ and $w^{\prime}=p_{j} c q_{j} c r_{n}=z l c u_{1} c r u_{1}^{\prime \prime} c r_{n} \in A^{*} u y A^{*}$. Clearly, the length of the derivation $w_{0} \stackrel{*}{\rightarrow} w_{j}=p_{j} c q_{j} c r_{j} \stackrel{*}{\rightarrow} w^{\prime}=p_{j} c q_{j} c r_{n}$ is strictly smaller than $n$.

Lemma 6.2. $S^{-1}\left(A^{*} u\right) \backslash A^{*} c u_{1} c r \subseteq S_{\infty}$.

Proof: Let $w \in S^{-1}\left(A^{*} u\right) \backslash A^{*} c u_{1} c r$. Then there exists a derivation: $w_{0} \rightarrow w_{1} \stackrel{*}{\rightarrow} w_{i} \stackrel{*}{\rightarrow} w_{n} \in$ $A^{*} u$. Let $j$ be the greatest index such that $w_{j} \notin A^{*} c u_{1} c r$. Then $w_{j}=p_{j} u u_{1}^{\prime \prime} c r \in A^{*} u y$, and it follows $w \in S^{-1}\left(A^{*} u y\right) \subseteq S_{\infty}$. 
Let us denote $F=\left\{c v_{1}\right\} \cup\left\{c u_{1} c r z \quad \mid v_{1}=r e z\right\}$. Remark that $F$ is finite and $S^{-1}\left(A^{*} l\right) F \subseteq$ $S^{-1}\left(A^{*} x l\right)$.

Lemma 6.3. $L_{x l} \subseteq L_{l} F$.

Proof: Let $w \in L_{x l}$. Then $w \stackrel{*}{\rightarrow} z x l=z l c v_{1}$. Let us consider two cases:

1. If $w=w^{\prime} c v_{1}$, we can deduce that $w^{\prime} \stackrel{*}{\rightarrow} z l$, so $w^{\prime} \in S^{-1}\left(A^{*} l\right)$. Since $w=w^{\prime} c v_{1} \notin S_{\infty}$, it follows $w^{\prime} \notin S_{\infty}$ and since $w \notin A S^{-1}\left(A^{*} x l\right)$, it follows $w^{\prime} \notin A S^{-1}\left(A^{*} l\right)$. Then $w^{\prime} \in L_{l}$ and $w=w^{\prime} c v_{1} \in L_{l} F$.

2. Else $w=w^{\prime} c r z$ with $z \in B^{*}$ and $r z \neq v_{1}$. Then $w^{*} \rightarrow p u z \rightarrow p v z \stackrel{*}{\rightarrow} p^{\prime} c v_{1}$. We show that $r e z=v_{1}$. Indeed, if it is not the case, pslcv $v_{1} c r \in S^{-1}\left(A^{*} u\right) \backslash A^{*} c u_{1} c r \subseteq S_{\infty}$ which implies $p v z \in S_{\infty}$ and $w \in S_{\infty}$. Since $w=w^{\prime} c r z \stackrel{*}{\rightarrow} p u z$, it follows $w^{\prime} c r \in S^{-1}\left(A^{*} u\right) \backslash S_{\infty} \subseteq$ $A^{*} c u_{1} c r$, so $w^{\prime} c r z=w^{\prime \prime} c u_{1} c r z \stackrel{*}{\rightarrow} p v z \in A^{*} x l$. Finally $w^{\prime \prime} \in S^{-1}\left(A^{*} l\right), w^{\prime \prime} \notin S_{\infty}$ and $w^{\prime \prime} \notin A S^{-1}\left(A^{*} l\right)$ which implies $w^{\prime \prime} \in L_{l}$ and $w \in L_{l} F$.

Let us denote $H=\{z \mid l \in \mathrm{RF}(r e z) \backslash \mathrm{RF}(r z)\}$. Remark that $H$ is finite and $S^{-1}\left(A^{*} u\right) H \subseteq$ $S^{-1}\left(A^{*} l\right)$.

Lemma 6.4. $L_{l} \subseteq l+L_{u} H$.

Proof: Let $w \in L_{l}$. If $w \in A^{*} l$ then $w=l$ otherwise $w=w^{\prime} c r z$ with $l \notin \operatorname{RF}(r z)$. Then there exists a derivation $w \stackrel{*}{\rightarrow} p u z \rightarrow p v z$ with $p v z \in A^{*}$ cre $z \cap S^{-1}\left(A^{*} l\right)$. Suppose that $l \notin \mathrm{RF}(r e z)$, then sslcv $_{1} c r \in S^{-1}\left(A^{*} u\right) \backslash A^{*} c u_{1} c r$ and it follows from the lemma 6.2 that $p v z$, and thus also $w$, is in $S_{\infty}$. This contradiction implies $l \in \operatorname{RF}(r e z)$ therefore $z \in H$ and $w^{\prime} c r \in S^{-1}\left(A^{*} u\right)$. Moreover $w \notin S_{\infty}$ and $w \notin A S^{-1}\left(A^{*} l\right)$ imply that $w^{\prime} c r \notin S_{\infty}$ and $w^{\prime} c r \notin A S^{-1}\left(A^{*} u\right)$. Finally $w^{\prime} c r \in L_{u}$ and $w \in L_{u} H$.

Lemma 6.5. $L_{u} \subseteq L_{l} c u_{1} c r$.

Proof: Let $w \in L_{u} \subseteq S^{-1}\left(A^{*} u\right) \backslash S_{\infty} \subseteq A^{*} c u_{1} c r$. Then $w=w^{\prime} c u_{1} c r \stackrel{*}{\rightarrow} w^{\prime \prime} c u_{1} c r$ with $w^{\prime \prime} \in A^{*} l$. It follows that $w^{\prime} \in S^{-1}\left(A^{*} l\right)$. Moreover $w^{\prime} \notin S_{\infty}$ and $w^{\prime} \notin A S^{-1}\left(A^{*} l\right)$ that imply $w^{\prime} \in L_{l}$ and $w \in L_{l} c u_{1} c r$.

Since, clearly, $S^{-1}\left(A^{*} l\right) c u_{1} c r H \subseteq S^{-1}\left(A^{*} l\right)$, it follows from the lemmata 6.4 and 6.5 that $L_{l} \subseteq$ $l\left(c u_{1} c r H\right)^{*} \subseteq S^{-1}\left(A^{*} l\right)$. Then, from the lemma 6.3, we get $L_{x l} \subseteq l\left(c u_{1} c r H\right)^{*} F \subseteq S^{-1}\left(A^{*} l\right) F \subseteq$ $S^{-1}\left(A^{*} x l\right)$ :

Lemma 6.6. $\quad L_{l} \subseteq l\left(c u_{1} c r H\right)^{*} \subseteq S^{-1}\left(A^{*} l\right)$ and $L_{x l} \subseteq l\left(c u_{1} c r H\right)^{*} F \subseteq S^{-1}\left(A^{*} x l\right)$.

Symmetrically, denoting $F^{\prime}=\left\{v_{1} c\right\} \cup\left\{z l c u_{1} c \mid v_{1}=z s l\right\}$ and $H^{\prime}=\{z \mid r \in \operatorname{LF}(z s l) \backslash \operatorname{LF}(z l)\}$, we get: 
Lemma 6.7. $R_{r} \subseteq\left(H^{\prime} l c u_{1} c\right)^{*} r \subseteq S^{-1}\left(r A^{*}\right)$ and $R_{r y} \subseteq F^{\prime}\left(H^{\prime} l c u_{1} c\right)^{*} r \subseteq S^{-1}\left(r y A^{*}\right)$.

Finally we obtain the following regular expression for $S_{\infty}$ :

Proposition 6.1. $S_{\infty}=A^{*} l\left(c u_{1} c r H\right)^{*}\left(F c u_{1} c+c u_{1} c F^{\prime}\right)\left(H^{\prime} l c u_{1} c\right)^{*} r A^{*}$

Proof: From the lemmata 6.1, 6.6 and 6.7, we obtain the following inclusions:

$$
\begin{gathered}
S_{\infty}=A^{*} S_{\infty}^{\min } A^{*} \subseteq A^{*}\left(L_{x l} c u_{1} c R_{r} \cup L_{l} c u_{1} c R_{r y}\right) A^{*} \\
\subseteq A^{*}\left(l\left(c u_{1} c r H\right)^{*} F c u_{1} c\left(H^{\prime} l c u_{1} c\right)^{*} r+l\left(c u_{1} c r H\right)^{*} c u_{1} c F^{\prime}\left(H^{\prime} l c u_{1} c\right)^{*} r\right) A^{*} \\
\subseteq A^{*}\left(S^{-1}\left(A^{*} x l\right) c u_{1} c S^{-1}\left(r A^{*}\right) \cup S^{-1}\left(A^{*} l\right) c u_{1} c S^{-1}\left(r y A^{*}\right)\right) A^{*} \\
\subseteq A^{*}\left(S^{-1}\left(A^{*}(x u+u y) A^{*}\right)\right) A^{*}=S_{\infty}
\end{gathered}
$$

Example 6.1. Let $S=\left\{a^{2} c a^{5} c \rightarrow a^{4} c a^{3} c a^{2}\right\}$ that was used in the example 3.2. Here $s=e=$ $l=a^{2}$ and $r=\varepsilon$. Recall that $x=a^{2} c a$ and $y=a^{3} c$. We deduce $F=c a^{3}+c a^{5} c a, H=\varepsilon+a$, $F^{\prime}=a^{3} c$ and $H^{\prime}=\emptyset$. Then

$$
S_{\infty}=(a+c)^{*} a^{2}\left(c a^{5} c+c a^{5} c a\right)^{*}\left[\left(c a^{3}+c a^{5} c a\right) c a^{5} c+c a^{5} c a^{3} c\right](a+c)^{*}
$$

\section{Conclusion and open questions}

This paper deals with the family $\mathcal{S}_{\text {grid }}$ of one-rule semi-Thue systems that are a borderline case for termination. Some of the results obtained here are shown to be false for arbitrary one-rule semi-Thue systems. For instance, it is proved in the section 4 that, for $S$ in $\mathcal{S}_{\text {grid }}$, $\operatorname{Loop}(S)$ is a constructible regular language whereas for $S=\{c \rightarrow c a c a\}, \operatorname{Loop}(S)$ is not regular. It is also proved that, for $S$ in $\mathcal{S}_{\text {grid }}, S(w)$ is always a context-free language whereas for $S=\left\{b a \rightarrow a^{2} b^{2}\right\}, S\left(b^{2} a^{2}\right)$ is not context-free [12]. At the contrary, some other results obtained here have been already shown to be true for other particular classes of one-rule semi-Thue systems. So we give new arguments in favour of some conjectures concerning arbitrary one-rule semi-Thue systems. For instance, it is proved that, for $S$ in $\mathcal{S}_{\text {grid }}, S_{\infty}=S^{-1}\left(A^{*} \operatorname{Loop}(S) A^{*}\right)$. This equality appears already in [15] and [23] for other subclasses of one-rule semi-Thue systems. So we can think :

Conjecture 1. $S_{\infty}=S^{-1}\left(A^{*} \operatorname{Loop}(S) A^{*}\right)$ for any one-rule semi-Thue system $S$.

Note that the validity of this conjecture would imply that if $S$ is a nonterminating one-rule semi-Thue system, then $\operatorname{Loop}(S)$ is not empty. Moreover, it would give a way to decide whether a word initiates an infinite derivation or not, as mentioned in [7]. For $S$ in $\mathcal{S}_{\text {grid }}$, it is proved that $A^{*} \operatorname{Loop}(S) A^{*}$, the ideal generated by $\operatorname{Loop}(S)$, is finitely generated. Does this property hold for arbitrary onerule Thue systems? It is also proved here that, for $S=\{u \rightarrow v\}$ with $|u|_{c}=|v|_{c}=2, S_{\infty}$ is a constructible regular language. This result was previously proved in [20] by Géraud Sénizergues for one-rule semi-Thue system with a left hand-side in $0^{*} 1^{*}$. So we can hope that :

Conjecture 2. If $S$ is a one-rule semi-Thue system, then $S_{\infty}$ is a constructible regular language. 
This property would imply the decidability of the (uniform) termination problem. However, remark that these two conjectures do not give a way to decide whether or not a word is in $\operatorname{Loop}(S)$.

Another possible extension of our results deals with one-rule grid Thue systems. For $S$ in $\mathcal{S}_{\text {grid }}$, one can consider the Thue system $\hat{S}=S \cup S^{-1}$ and ask: Is it true that $\forall w, \hat{S}(w)$ is a context-free language? Is it true that if $S$ and $S^{-1}$ are terminating then $\forall w, \hat{S}(w)$ is a finite language? Remark that this last question can be also raised when $S$ is an arbitrary one-rule Thue system.

\section{References}

[1] Anne-Cécile Caron. Linear bounded automata and rewrite systems: Influence of initial configurations on decision properties. In TAPSOFT, Vol.1, pages 74-89, 1991.

[2] Max Dauchet. Termination of rewriting is undecidable in the one-rule case. In MFCS, pages 262-270, 1988.

[3] Nachum Dershowitz. Termination of rewriting. J. Symb. Comput., 3(1/2):69-116, 1987.

[4] Alfons Geser. Decidability of termination of grid string rewriting rules. SIAM J. Comput., 31(4):1156-1168, 2002.

[5] Alfons Geser. Loops of superexponential lengths in one-rule string rewriting. In RTA, pages 267-280, 2002.

[6] Alfons Geser. Termination of string rewriting rules that have one pair of overlaps. In RTA, pages 410-423, 2003.

[7] Alfons Geser and Hans Zantema. Non-looping string rewriting. ITA, 33(3):279-302, 1999.

[8] Seymour Ginsburg. The Mathematical Theory of Context-Free Languages. McGraw-Hill, Inc., New York, NY, USA, 1966.

[9] Seymour Ginsburg and Edwin H. Spanier. Bounded algol-like languages. Transactions of the American Mathematical Society, 113(2):333-368, 1964.

[10] John V. Guttag, Deepak Kapur, and David R. Musser. On proving uniform termination and restricted termination of rewriting systems. SIAM J. Comput., 12(1):189-214, 1983.

[11] Winfried Kurth. One-rule semi-thue systems with loops of length one, two or three. ITA, 30(5):415-429, 1996.

[12] Michel Latteux and Yves Roos. The image of a word by a one-rule semi-Thue system is not always context-free. http://www.lifl.fr/ ${ }^{\sim}$ yroos/al/one-rule-context-free.pdf, 2011.

[13] Michel Latteux and Yves Roos. A new proof for the uniform termination problem of one-rule grid semi-Thue systems. http://www.lifl.fr/ yroos/al/one-rule-utermination.pdf, 2011.

[14] Yuri Matiyasevich and Géraud Sénizergues. Decision problems for semi-thue systems with a few rules. In LICS, pages 523-531, 1996.

[15] Robert Mcnaughton. Well behaved derivations in one-rule semi-thue systems. Technical Report 95-15, Dept. of Computer Science, Rennsselaer Polytechnic Institute, 1995.

[16] Robert Mcnaughton. Semi-thue systems with an inhibitor. J. Autom. Reason., 26(4):409-431, 2001.

[17] Wojciech Moczydlowski and Alfons Geser. Termination of single-threaded one-rule semi-thue systems. In RTA, pages 338-352, 2005. 
[18] Masahiko Sakai and Yi Wang. Undecidable properties on length-two string rewriting systems. Electronic Notes in Theoretical Computer Science, 204:53 - 69, 2008. Proceedings of the 7th International Workshop on Reduction Strategies in Rewriting and Programming (WRS 2007).

[19] Géraud Sénizergues. Some undecidable termination problems for semi-thue systems. Theor. Comput. Sci., 142(2):257-276, 1995.

[20] Géraud Sénizergues. On the termination problem for one-rule semi-thue system. In RTA, pages 302-316, 1996.

[21] Celia Wrathall. Confluence of one-rule thue systems. In IWWERT '90: Proceedings of the First International Workshop on Word Equations and Related Topics, pages 237-246, London, UK, 1992. Springer-Verlag.

[22] Wang Yi, Sakai Masahiko, and Nishida Naoki. Confluence of length preserving string rewriting systems is undecidable(theory of computer science and its applications). RIMS Kokyuroku, 1554:171-177, 2007.

[23] Hans Zantema and Alfons Geser. A complete characterization of termination of $0^{\mathrm{p}}{ }_{1} \mathrm{q}_{-}>1^{\mathrm{r}}$ $0^{\mathrm{S}}$. Appl. Algebra Eng. Commun. Comput., 11(1):1-25, 2000. 